\title{
Positiveness and Observer-Based Finite-Time Control for a Class of Markov Jump Systems with Some Complex Environment Parameters
}

\author{
Chengcheng Ren $\mathbb{D}^{1,2}$ and Shuping He $\mathbb{D}^{1,2}$ \\ ${ }^{1}$ Key Laboratory of Intelligent Computing \& Signal Processing (Ministry of Education), School of Electrical Engineering \& Automation, \\ Anhui University, Hefei 230601, China \\ ${ }^{2}$ Institute of Physical Science and Information Technology, Anhui University, Hefei 230601, China
}

Correspondence should be addressed to Shuping He; shuping.he@ahu.edu.cn

Received 17 August 2018; Accepted 23 October 2018; Published 8 November 2018

Guest Editor: Junpei Zhong

Copyright ( 2018 Chengcheng Ren and Shuping He. This is an open access article distributed under the Creative Commons Attribution License, which permits unrestricted use, distribution, and reproduction in any medium, provided the original work is properly cited.

\begin{abstract}
An observer-based finite-time $L_{2}-L_{\infty}$ control law is devised for a class of positive Markov jump systems in a complex environment. The complex environment parameters include bounded uncertainties, unknown nonlinearities, and external disturbances. The objective is to devise an appropriate observer-based control law that makes the corresponding augment error dynamic Markov jump systems be positive and finite-time stabilizable and satisfy the given $L_{2}-L_{\infty}$ disturbance attenuation index. A sufficient condition is initially established on the existence of the observer-based finite-time controller by using proper stochastic Lyapunov-Krasovskii functional. The design criteria are presented by means of linear matrix inequalities. Finally, the feasibility and validity of the main results can be illustrated through a numerical example.
\end{abstract}

\section{Introduction}

As a special kind of hybrid systems, Markov jump systems (MJSs) consist of two kinds of hybrid dynamic forms. One form is characterized by a discrete state and continuous-time Markov process, called mode; the other form is described by state space equations in each mode, called state. This kind of MJSs was firstly proposed by Krasovskii and Lidskii [1] in 1960s. Due to the stochastic Markov process, it is always considered as a special stochastic system. Moreover, MJSs tend to describe systems in which structures are subjected to abrupt stochastic variations. Such variations usually come from sudden failure of connection between system components, abrupt environmental changes, or changes in the operating point of nonlinear dynamics. MJSs are widely used in many applications, for example, economic systems [2], power electronic system [3], communication systems [4], and circuit network systems [5]. Due to the wide applications, the research of MJSs has been paid much attention in the past decades. For more details about this issue, we can refer to [6$15]$.
On another research front, we notice that in some dynamic systems there always exist nonnegative characteristics, such as the number of animals, absolute temperature, density of matter, and the concentration of chemical reactions [16-18]. To describe these characteristics, we usually use a positive system to illustrate the nonnegative (positive, strictly) dynamic behavior of state variables. Positive system is a special system; the states and the outputs are both nonnegative (positive, strictly) for any nonnegative (positive, strictly) initial conditions. More recently, the research of positive system has become a heated topic and many publications about this issue have been developed; see, for example, [19$23]$ and the references therein. However, the main result in above references only considered that the states of the systems can be measurable case. It should be noted that the states always cannot be measurable in some practical application systems [24-26]. Up to present, the observerbased finite-time control problem of positive Markov jump systems (PMJSs) with some complex environment parameters has not been intensively studied, saying nothing of the simultaneous presence of uncertainties [27-32], unknown 
nonlinearities [21, 33-37], and external disturbances [38-40]. These motivate our research on observer-based finite-time controller design problem for MJSs.

This paper analyzes the problems of observer-based finite-time $L_{2}-L_{\infty}$ control for a class of PMJSs in a complex environment. Compared with existing results related to Markovian jump systems, the main contributions and difficulties are addressed as follows.

(i) The main contributions of this paper mainly consist of three aspects. Firstly, we aim to analyze the stabilizable problem of a class of PMJSs with some complex environment parameters. Secondly, we try to design an appropriate observer-based finite-time $L_{2}-L_{\infty}$ control law to ensure that closed-loop Markov jump systems be finite-time stabilizable when the states of the system cannot be measured. Thirdly, we attempt to give a sufficient condition to guarantee the positiveness of the augment error dynamic MJSs and we essay to illustrate the validity of the designed method through a numerical example.

(ii) Different from the existing results in $[3,6,12,13]$, the main difficulties of the paper are how to design an appropriate control law for PMJSs in a complex environment with nonmeasurable states such that the corresponding augment error dynamic Markov jump systems be positive and finitetime stabilizable and satisfy the given $L_{2}-L_{\infty}$ disturbance attenuation index. It is necessary to point out that the main results in $[3,6,12,13]$ only considering the states of the systems can be measurable case.
In this paper, all matrices are assumed with proper dimensions and all notations are quite standard. The implication of the symbols is given in Table 1 .

\section{Preliminaries}

2.1. System Description. For a probability space $\Theta_{s}:\left(\Phi, \Lambda, \prod_{r}\right)$ composed of sample space $\Phi$, algebra of events $\Lambda$, and the probability measure $\Pi_{r}$ which is defined on $\Lambda$, assume that the stochastic process $\left\{r_{t}, t \geq 0\right\}$ is a continuoustime discrete-state Markov stochastic process in a finite set $M=\{1,2, \ldots, N\}$ over the probability space $\Theta_{s}$. The transition probability matrix $\prod_{r}=\left\{\prod_{i j}(t), i, j \in M\right\}$ is defined as

$$
\begin{aligned}
\prod_{i j}(t) & =\prod\left\{r_{t+\Delta t}=j \mid r_{t}=i\right\} \\
& = \begin{cases}\pi_{i j} \Delta t+o(\Delta t), & i \neq j, \\
1+\pi_{i i} \Delta t+o(\Delta t), & i=j\end{cases}
\end{aligned}
$$

where $\Delta t>0$ and $\lim _{\Delta t \longrightarrow 0}(o(\Delta t) / \Delta t) \longrightarrow 0 ; \pi_{i j}(t) \geq 0$ is the transition probability rate from mode $i$ at time $t$ to mode $j$ at time $t+\Delta t$ and satisfies $\sum_{j=1, i \neq j}^{N} \pi_{i j}=-\pi_{i i}$.

In this paper, we investigate a class of PMJSs in complex environments including uncertainties, unknown nonlinearities, and unknown external disturbances. The PMJSs in the probability space $\Theta_{s}$ are described by

$$
\sum_{S}:\left\{\begin{array}{l}
\dot{x}(t)=\left[A\left(r_{t}\right)+A_{\Delta}\left(r_{t}, t\right)\right] x(t)+\left[B\left(r_{t}\right)+B_{\Delta}\left(r_{t}, t\right)\right] u(t)+F_{1}\left(r_{t}\right) f(x(t), t)+W_{1}\left(r_{t}\right) w(t) \\
y(t)=\left[C\left(r_{t}\right)+C_{\Delta}\left(r_{t}, t\right)\right] x(t) \\
m(t)=\left[M\left(r_{t}\right)+M_{\Delta}\left(r_{t}, t\right)\right] x(t)+W_{2}\left(r_{t}\right) w(t) \\
x(0)=x_{0} \\
r(0)=r_{0} \\
t=0
\end{array}\right.
$$

where $x(t) \in R^{n}$ denote the state, $m(t) \in R^{l}$ denote the measured output, $y(t) \in R^{m}$ denote the controlled output, $w(t) \in L_{2}^{P}[0,+\infty]$ denote the unknown external disturbance, $u(t) \in R^{q}$ denote the controlled input, and $f(x(t), t)$ denote the unknown nonlinearities, which indicate the nonlinear disturbances related to the state. $r_{0}$ and $x_{0}$ are initial mode and initial state, respectively. $\left[A\left(r_{i}\right)+A_{\Delta}\left(r_{i}, t\right)\right]$ is a mode-dependent Metzler matrix; $B\left(r_{i}\right), B_{\Delta}\left(r_{i}, t\right), F\left(r_{i}\right), W_{1}\left(r_{i}\right), W_{2}\left(r_{i}\right), C\left(r_{i}\right), C_{\Delta}\left(r_{i}, t\right), M\left(r_{i}\right)$, and $M_{\Delta}\left(r_{i}, t\right)$ are mode-dependent positive matrices. For convenience, we use $A_{i}, A_{\Delta i}, B_{i}, B_{\Delta i}, F_{1 i}, W_{1 i}, W_{2 i}, C_{i}, C_{\Delta i}$, $M_{i}$, and $M_{\Delta i}$ to denote the relevant parameter matrices with $r_{t}=i$. Moreover, the time-varying uncertain matrices satisfy

$$
\left[\begin{array}{llll}
A_{\Delta i} & B_{\Delta i} & C_{\Delta i} & M_{\Delta i}
\end{array}\right]=L_{1 i} \Gamma_{i}(t)\left[\begin{array}{llll}
N_{1 i} & N_{2 i} & N_{3 i} & N_{4 i}
\end{array}\right]
$$

where $\Gamma_{i}(t)$ is a mode-dependent Lebesgue norm measurable function and satisfies $\left\|\Gamma_{i}(t)\right\| \leq 1 ; L_{1 i}, N_{1 i}, N_{2 i}, N_{3 i}$, and $N_{4 i}$ are known mode-dependent matrices.

Remark 1. In this paper, the uncertain matrices $A_{\Delta i}, B_{\Delta i}, C_{\Delta i}$, and $M_{\Delta i}$ in (3) can be considered as admissible conditions. In actual applications, it is usually impossible directly to get the accurate mathematical model of realistic dynamics because of some complex environment including unknown nonlinearities, environmental noises, and time-varying parameters $[10,27]$. Thus, the uncertain dynamics existing in PMJSs (2) reflect the inexactness in mathematical modeling of such Markov jump systems. Moreover, the mode-dependent Lebesgue norm measurable function $\Gamma_{i}(t)$ is selected as a full row rank matrix and it also can be considered as statedependent; that is, $\Gamma_{i}(t)=\Gamma_{i}(t, x(t))$ if $\left\|\Gamma_{i}(t, x(t))\right\| \leq 1$. For more results of this issue, we refer readers to [30-32]. 
TABLE 1: Symbols throughout this paper.

\begin{tabular}{lccc}
\hline Symbol & Means & Symbol & Means \\
\hline$A^{\mathrm{T}}$ & matrix transpose & $\operatorname{diag}\{A \quad B\}$ & block-diagonal matrix of $A$ and $B$ \\
$A^{-1}$ & matrix inverse & $\lambda_{\min (\max )}(D)$ & minimum (maximum) eigenvalue of $D$ \\
$\|\cdot\|$ & Euclidean vector norm & 0 & $n \times m$ real matrices \\
$I$ & $\quad$ unit matrix & $\mathfrak{R}^{n \times m}$ & zero matrix \\
$\mathfrak{R}^{n}$ & $n$-dimensional Euclidean space & $H \succ(<, \geq, \preceq) 0$ & all elements of the matrix $H$ are positive (negative, non-negative, non-positive) \\
$*$ & Symmetric matrix & $P>(<, \geq, \leq) 0$ & positive- (negative, non-negative, non-positive) definite matrix \\
\hline
\end{tabular}

When the states of PMJSs (2) cannot be available, we can construct the following state observer and feedback control law:

$$
\begin{aligned}
& \bar{\Sigma}_{s}: \\
& \qquad \begin{array}{l}
\dot{\bar{x}}=A_{i} \bar{x}(t)+\left(B_{i}+B_{\Delta i}\right) u(t)+E_{i}[m(t)-\bar{m}(t)] \\
\bar{m}(t)=M_{i} \bar{x}(t) \\
u(t)=K_{i} \bar{x}(t) \\
\bar{y}(t)=C_{i} \bar{x}(t) \\
x(0)=x_{0} \\
r(0)=r_{0} \\
t=0
\end{array}
\end{aligned}
$$

where $\bar{x}(t) \in R^{n}$ denote the estimated state; $\bar{m}(t) \in R^{l}$ denote the observer output; $\bar{y}(t) \in R^{m}$ denote the estimation output. $E_{i}$ and $K_{i}$ are observer and control law gains to be devised, respectively. The state estimated error and the controlled output error of $\Sigma_{s}$ are defined by $e(t)=x(t)-\bar{x}(t)$ and $z(t)=$ $y(t)-\bar{y}(t)$, respectively. Therefore, we have the following MJSs by (2) and (4):

$$
\begin{aligned}
\dot{x}(t)= & {\left[\left(A_{i}+A_{\Delta i}\right)+\left(B_{i}+B_{\Delta i}\right) K_{i}\right] x(t) } \\
& -\left(B_{i}+B_{\Delta i}\right) K_{i} e(t)+F_{1 i} f(x(t), t) \\
& +W_{1 i} w(t) \\
\dot{e}(t)= & \left(A_{\Delta i}-E_{i} M_{\Delta i}\right) x(t)+\left(A_{i}-E_{i} M_{i}\right) e(t) \\
& +F_{1 i} f(x(t), t)+\left(W_{1 i}-E_{i} W_{2 i}\right) w(t)
\end{aligned}
$$

Letting $\widetilde{x}(t)=\operatorname{col}[x(t) e(t)]$, the closed-loop augment error dynamic MJSs can be represented as

$$
\widetilde{\Sigma}_{s}:\left\{\begin{array}{l}
\dot{\tilde{x}}=\left(\widetilde{A}_{i}+\widetilde{A}_{\Delta i}\right) \widetilde{x}(t)+\widetilde{F}_{i} f(\widetilde{x}(t), t)+\widetilde{W}_{i} w(t) \\
z(t)=\widetilde{C}_{i} \widetilde{x}(t) \\
\widetilde{x}(0)=\widetilde{x}_{0}, \\
t=0
\end{array}\right.
$$

where

$$
\begin{aligned}
\widetilde{A}_{i} & =\left[\begin{array}{cc}
A_{i}+B_{i} K_{i} & -B_{i} K_{i} \\
0 & A_{i}-E_{i} M_{i}
\end{array}\right], \\
\widetilde{A}_{\Delta i} & =\left[\begin{array}{cc}
A_{\Delta i}+B_{\Delta i} K_{i} & -B_{\Delta i} K_{i} \\
A_{\Delta i}-E_{i} M_{\Delta i} & 0
\end{array}\right], \\
\widetilde{F}_{i} & =\left[\begin{array}{l}
F_{1 i} \\
F_{1 i}
\end{array}\right], \\
\widetilde{W}_{i} & =\left[\begin{array}{c}
W_{1 i} \\
W_{1 i}-E_{i} W_{2 i}
\end{array}\right], \\
\widetilde{C}_{i} & =\left[\begin{array}{ll}
C_{\Delta i} & C_{i}
\end{array}\right], \\
f(x(t), t) & =f(\tilde{x}(t), t) .
\end{aligned}
$$

2.2. Main Definitions, Lemmas, and Assumptions. The following main definitions, lemmas, and assumptions are important for analyzing and giving the main results of the paper.

Definition 2. For given constants $T>0$ and $\mu_{1}>0$, the augment error dynamic MJSs (6) are finite-time stabilizable (FTS) with regard to $\left(\mu_{1}, \mu_{2}, T, R\right)$, if there exist constants $u_{2}>u_{1}>0$ and positive-definite matrix $R>0$, such that

$$
\begin{aligned}
\mathrm{E}\left\{\tilde{x}^{\mathrm{T}}(t) R \tilde{x}(t)\right\}< & \mu_{2}, \\
& \forall t \in\left[\begin{array}{ll}
0 & T
\end{array}\right], \text { if } \widetilde{x}^{\mathrm{T}}(0) R \tilde{x}(0) \leq \mu_{1} .
\end{aligned}
$$

Definition 3. For given constants $T>0$ and $\mu_{1}>0, \bar{\Sigma}_{s}$ is said to be the finite-time $L_{2}-L_{\infty}$ observer-based state feedback control law of MJSs (6) under the zero initial condition, if the augment error dynamic MJSs (6) are FTS with regard to $\left(\mu_{1}, \mu_{2}, T, R\right)$ and satisfy

$$
\mathrm{E}\left\{\|z(t)\|_{\infty}^{2}\right\}-\delta^{2}\|w(t)\|_{2}^{2}<0
$$

where $d>0, \delta>0, \mathrm{E}\left\{\|z(t)\|_{\infty}^{2}\right\}=E\left\{\sup _{t \in[0 T]}\left[z(t)^{\mathrm{T}} z(t)\right]\right\}$, and $\|w(t)\|_{2}^{2}=\int_{0}^{T} w(t)^{\mathrm{T}} w(t) d t$. 
Definition 4. The weak infinitesimal operator of stochastic Lyapunov-Krasovskii functional (SLKF) $V[\tilde{x}(t), i, t>0]$ is defined as

$$
\begin{aligned}
& \mathfrak{I} V[\tilde{x}(t), i, t] \\
& =\lim _{\Delta t \rightarrow 0} \frac{1}{\Delta t}\left[E \left\{V\left[\tilde{x}(t+\Delta t), r_{(t+\Delta t)}, t+\Delta t\right] \mid \tilde{x}(t),\right.\right. \\
& \left.\left.\quad r_{t}\right\}-V\left[\tilde{x}(t), r_{t}, t\right]\right]=\frac{\partial}{\partial t} V[\tilde{x}(t), i, t]+\frac{\partial}{\partial \widetilde{x}} \\
& \quad V[\tilde{x}(t), i, t] \dot{\tilde{x}}(t)+\sum_{j=1}^{N} \pi_{i j} V[\tilde{x}(t), j, t]
\end{aligned}
$$

Definition 5. MJSs (2) are positive, if, for the initial conditions $x_{0}>0$ and $r_{0}>0$ and the controlled input $u(t)>0$, the relevant trajectories of $\Sigma_{s}$ satisfy $x(t)>0$ and $y(t)>0, \forall t>$ 0 .

Lemma 6 (see [41]). $A_{i}+A_{\Delta i}$ is said to be a Metzler matrix, if there exists a mode-dependent constant $\varepsilon_{i}$ satisfying $A_{i}+A_{\Delta i}+$ $\varepsilon_{i} I \geq 0$, where $A_{i}+A_{\Delta i}$ is a real square matrix.

Lemma 7 (see [41]). MJSs (2) are said to be positive if and only if $A_{i}+A_{\Delta i}$ is a Metzler matrix and $B_{i}+B_{\Delta i} \succeq 0, F_{i} \succeq 0, W_{1 i} \succeq$ $0, C_{i}+C_{\Delta i} \succeq 0, M_{i}+M_{\Delta i} \succeq 0$, and $W_{2 i} \succeq 0$.

Lemma 8 (see [30]). Suppose that $L_{i}$ and $N_{i}$ are modedependent real matrices and $\Gamma_{i}(t)$ is a mode-dependent Lebesgue norm measurable function and satisfies $\left\|\Gamma_{i}(t)\right\| \leq 1$. There exists a mode-dependent constant $\alpha_{i}>0$, satisfying

$$
L_{i} \Gamma_{i}(t) N_{i}+\left[L_{i} \Gamma_{i}(t) N_{i}\right]^{\mathrm{T}}<\alpha_{i}^{-1} L_{i} L_{i}^{\mathrm{T}}+\alpha_{i} N_{i}^{\mathrm{T}} N_{i}
$$

Lemma 9 (see [30]). Suppose that $F_{i}$ and $E_{i}$ are modedependent real matrices. There exists a positive-definite matrix $M$ and $a$ mode-dependent constant $\beta_{i}>0$, satisfying

$$
F_{i}^{\mathrm{T}} E_{i}+E_{i}^{\mathrm{T}} F_{i} \leq \beta_{i} F_{i}^{\mathrm{T}} M F_{i}+\beta^{-1} E_{i}^{\mathrm{T}} M^{-1} E_{i} .
$$

Assumption 10. The mode-dependent nonlinear function $f(x(t), t)$ satisfies the following Lipschitz condition:

$$
\|f(x(t), t)\| \leq\|G \quad x(t)\|
$$

where $G$ is a real matrix with proper dimension.

Assumption 11. For given constant $d>0, w(t)$ is energybounded and satisfies

$$
\int_{0}^{\mathrm{T}} w^{\mathrm{T}}(t) w(t) d t \leq d
$$

Remark 12. Assumption 10 guarantees that we can use linearization method to study the nonlinear systems by means of linear matrix inequalities $[3,5,11]$. In the design of observer-based finite-time $L_{2}-L_{\infty}$ control law, Assumption 11 is given to assume that the unknown external disturbance is to be an arbitrary deterministic signal of bounded energy $[20,24,27]$.

\section{Main Results}

3.1. FTS Analysis. In this subsection, the FTS analysis for the augment error dynamic MJSs (6) will be considered. Based on the SLKF approach and LMIs techniques, a sufficient condition of FTS will be given in Theorem 13.

Theorem 13. For given constants $T>0, \mu_{1}>0, \gamma>0$, and $d>0$, the augment error dynamic MJSs (6) are FTS with regard to $\left(\mu_{1}, \mu_{2}, T, R, \gamma\right)$, where $\mu_{2}>\mu_{1}>0$ and $R>0$, if there exists a set of mode-dependent positive-definite symmetric matrix $\widetilde{P}_{i} \in R^{n \times n}$, positive-definite symmetric matrix $R>0$, matrix $G \in \Re^{n \times n}$, a set of mode-dependent constant $\beta_{i}>0$, and constant $\gamma>0$, such that

$$
\begin{gathered}
{\left[\begin{array}{ccc}
\vartheta_{1}-\gamma \widetilde{P}_{i} & \widetilde{P}_{i} \widetilde{W}_{i} & \widetilde{P}_{i} \widetilde{F}_{i} \\
* & -I & 0 \\
* & * & -\beta_{i} I
\end{array}\right]<0} \\
\frac{e^{\gamma \mathrm{T}}\left[\lambda_{1} u_{1}+(d / r)\left(1-e^{-\gamma \mathrm{T}}\right)\right]}{\lambda_{2}}<u_{2}
\end{gathered}
$$

where $\vartheta_{1}=\left(\widetilde{A}_{i}+\widetilde{A}_{\Delta i}\right)^{\mathrm{T}} \widetilde{P}_{i}+\widetilde{P}_{i}\left(A_{i}+A_{\Delta i}\right)+\beta_{i} G^{\mathrm{T}} G+\sum_{j=1}^{N} \pi_{i j} \widetilde{P}_{j}$, $\lambda_{1}=\max _{r \in M} \lambda_{\max }\left[\bar{P}_{i}\right], \lambda_{2}=\min _{r \in M} \lambda_{\min }\left[\bar{P}_{i}\right]$, and $\bar{P}_{i}=$ $R^{-1 / 2} \widetilde{P}_{i} R^{-1 / 2}$.

Proof. See Appendix A.

3.2. Finite-Time $L_{2}-L_{\infty}$ Disturbance Attenuation Index Analysis. Theorem 13 gives a sufficient condition of FTS for augment error dynamic MJSs (6). Recalling Definition 3, we will give the following Theorem 14 to analyze the observerbased finite-time $L_{2}-L_{\infty}$ control law design.

Theorem 14. For given constants $T>0, \mu_{1}>0, \gamma>$ 0 , and $d>0$, the augment error dynamic MJSs (6) are FTS with regard to $\left(\mu_{1}, \mu_{2}, T, R, \gamma\right)$, where $\mu_{2}>\mu_{1}>0$ and satisfy the given $L_{2}-L_{\infty}$ disturbance rejection disturbance attenuation index (10), if there exists a set of mode-dependent positive-definite symmetric matrix $P_{i} \in R^{n \times n}$, positive-definite symmetric matrix $R>0$, matrix $G \in \mathfrak{R}^{n \times n}$, a set of modedependent constant $\beta_{i}>0$, and constant $\gamma>0$, such that inequalities (15)-(16) and the following relation hold:

$$
\left[\begin{array}{cc}
\widetilde{P}_{i} & \widetilde{C}_{i}^{\mathrm{T}} \\
* & \delta^{2} I
\end{array}\right]>0
$$

Proof. See Appendix B.

3.3. Positiveness and Observer-Based Control Law Gain Solution. Recalling Definition 5, following sufficient condition will be given to ensure the positiveness of the augment error dynamic MJSs (6) in Theorem 15. It should be noted that there exist some time-varying uncertain matrices in Theorem 13. Therefore, it is difficult to obtain the observer gain $E_{i}$ and the control law gain $K_{i}$ from matrix inequalities (15)-(17). It is necessary for us to convert the nonlinear matrix inequalities 
(15)-(17) into the solvable inequalities, which can be directly solved by Matlab LMI toolbox.

Theorem 15. For given constants $T>0, \mu_{1}>0, \gamma>$ 0 , and $d>0$, there exists a finite-time $L_{2}-L_{\infty}$ observerbased control law with $K_{i}=S_{i} V_{i}^{-1}$ and $E_{i}=U_{i} M_{i}^{\mathrm{T}}$ and the augment error dynamic MJSs (6) are positive and FTS and satisfy the given $L_{2}-L_{\infty}$ disturbance rejection disturbance attenuation index (9) with regard to $\left(\mu_{1}, \mu_{2}, T, R, \gamma\right)$, where $\mu_{2}>\mu_{1}>0$, if there exists a set of mode-dependent positive-definite symmetric matrix $U_{i} \in R^{n \times n}$, positive-definite symmetric matrix $R>0$, a set of mode-dependent matrices $S_{i} \in R^{p \times n}$ and $Q_{1 i} \in R^{1 \times n}$, a set of mode-dependent constants $\alpha_{1 i}>0, \alpha_{2 i}>0, \alpha_{3 i}>0, \alpha_{4 i}>0, \beta_{i}>0, \varepsilon_{i}>0$, and $v_{i}>0$, and constants $\mu_{2}>0, \delta>0, \lambda_{1}>0$, and $\lambda_{2}>0 \gamma>0$, such that

$$
\begin{aligned}
{\left[\begin{array}{ccc}
\bigsqcup_{11} & \bigsqcup_{22} \\
* & \bigsqcup_{22}
\end{array}\right] } & <0 \\
{\left[\begin{array}{ccccc}
-U_{i} & 0 & 0 & U_{i} N_{3 i}^{\mathrm{T}} & 0 \\
* & -U_{i} & -C_{i}^{\mathrm{T}} & 0 & 0 \\
* & * & -\delta^{2} I & 0 & L_{1 i} \\
* & * & * & -\alpha_{4 i}^{-1} & 0 \\
* & * & * & * & \alpha_{4 i}
\end{array}\right] } & <0 \\
{\left[\begin{array}{rlr}
d\left(1-e^{-\gamma \mathrm{T}}\right)-\gamma \lambda_{2} \mu_{2} e^{-\gamma \mathrm{T}} & \sqrt{\gamma \mu_{1}} \\
\sqrt{\gamma \mu_{1}} & -\lambda_{1}
\end{array}\right] } & <0 \\
\lambda_{2} R_{i}^{-1} & <U_{i}<R_{i}^{-1} \\
A_{i} U_{i}+B_{i} S_{i}+\varepsilon_{i} I & \succeq 0 \\
A_{i}-E_{i} M_{i}+v_{i} I & \succeq 0 \\
B_{i} S_{i} & \leq 0 \\
W_{1 i} U_{i}-M_{i}^{\mathrm{T}} W_{2 i} U_{i} & \succeq 0
\end{aligned}
$$

where

$$
\begin{aligned}
\coprod_{11} & =\left[\begin{array}{lllll}
\theta_{1} & \theta_{2} & \theta_{3} & \theta_{4} & \theta_{5} \\
* & \theta_{6} & \theta_{7} & \theta_{8} & \theta_{9} \\
* & * & \theta_{10} & \theta_{11} & \theta_{12} \\
* & * & * & \theta_{13} & \theta_{14} \\
* & * & * & * & \theta_{15}
\end{array}\right], \\
\coprod_{12} & =\left[\begin{array}{ccccc}
\varphi_{1} & \varphi_{2} & \varphi_{3} & \varphi_{4} & 0 \\
0 & 0 & \varphi_{7} & 0 & \varphi_{9} \\
0 & 0 & 0 & 0 & 0 \\
0 & 0 & 0 & 0 & 0 \\
0 & 0 & 0 & 0 & 0
\end{array}\right], \\
\theta_{5} & =\theta_{11}=\theta_{12}=\theta_{14}=0 \\
\coprod_{22} & =\operatorname{diag}\left\{-\beta_{i}^{-1} I-\alpha_{2 i}^{-1} I-\alpha_{1 i}^{-1} I-\alpha_{3 i}^{-1} I-\alpha_{3 i} I\right\}
\end{aligned}
$$

$$
\begin{aligned}
\theta_{1}= & A_{i} U_{i}+U_{i} A_{i}^{\mathrm{T}}+B_{i} S_{i}+S_{i}^{\mathrm{T}} B_{i}^{\mathrm{T}}+\left(\pi_{i i}-\gamma\right) U_{i} \\
& +\alpha_{1 i}^{-1} L_{1 i} L_{1 i}^{\mathrm{T}}, \\
\theta_{2}= & -B_{i} S_{i}, \\
\theta_{3}= & W_{1 i} U_{i}, \\
\theta_{4}= & F_{1 i} U_{i}, \\
\theta_{6}= & A_{i} U_{i}+U_{i} A_{i}^{\mathrm{T}}+\left(\pi_{i i}-\gamma\right) U_{i}+\alpha_{2 i}^{-1} L_{1 i} L_{1 i}^{\mathrm{T}}, \\
\theta_{7}= & W_{1 i} U_{i}-M_{i}^{\mathrm{T}} W_{2 i} U_{i}, \\
\theta_{8}= & F_{1 i} U_{i}, \\
\theta_{9}= & U_{i} M_{i}^{\mathrm{T}}, \\
\theta_{10}= & -I, \\
\theta_{13}= & -\beta_{i} I, \\
\theta_{15}= & -2 I, \\
\varphi_{1}= & U_{i} G_{i}^{\mathrm{T}}, \\
\varphi_{2}= & U_{i} N_{1 i}^{\mathrm{T}}, \\
\varphi_{3}= & U_{i} N_{1 i}^{\mathrm{T}}+S_{i}^{\mathrm{T}} N_{2 i}^{\mathrm{T}}, \\
\varphi_{4}= & U_{i} N_{4 i}^{\mathrm{T}}, \\
\varphi_{5}= & -S_{i}^{\mathrm{T}} N_{2 i}^{\mathrm{T}}, \\
\varphi_{6}= & Q_{1 i} .
\end{aligned}
$$

Proof. See Appendix C.

Corollary 16. The sufficient conditions to design the stochastic finite-time $L_{2}-L_{\infty}$ observer-based control law for a class of PMJSs in complex environments have been presented in Theorems 13-15. Considering that the coupling inequalities (18)-(25) are related to $U_{i}, S_{i}, \alpha_{1 i}, \alpha_{2 i}, \alpha_{3 i}, \alpha_{4 i}, \beta_{i}, \mu_{1}, \mu_{2}, T$, $d, \gamma$, and $\widetilde{\delta}=\delta \sqrt{e^{\gamma T}}$, we have the optimization algorithm by setting $\widetilde{\delta}$ as an optimization variable value:

$$
\begin{aligned}
\min _{U_{i}, S_{i}, \alpha_{1 i}, \alpha_{2 i}, \alpha_{3 i}, \alpha_{4 i}, \beta_{i}, \mu_{1}, \mu_{2}, \delta, T, d, \gamma} & \widetilde{\delta} \\
\text { s.t. } & \text { inequalities (18) - (25). }
\end{aligned}
$$

Remark 17. It should be pointed out that the optimization algorithm (27) is given to solve the unknown matrices and parameters through Matlab LMI toolbox. Recalling inequality (20), it is known that all of the parameters in $d\left(1-e^{-\gamma T}\right)-$ $\gamma \lambda_{2} \mu_{2} e^{-\gamma T}$ are linear and also can be solved through Matlab LMI toolbox by setting $\mu_{2}$ as an unknown parameter. Considering $L_{2}-L_{\infty}$ disturbance attenuation index in Definition 3, we select $\widetilde{\delta}=\delta \sqrt{e^{\gamma T}}$ as an optimization variable value in optimization algorithm (27). 
Remark 18. For PMJSs (2) in complex environments without uncertainties in probability space $\Theta_{s}$, we have the following

dynamic systems:

$$
\Sigma_{s}^{\prime}\left\{\begin{array}{l}
\dot{x}(t)=A\left(r_{t}\right) x(t)+B\left(r_{t}\right) u(t)+F_{1}\left(r_{t}\right) f(x(t), t)+W_{1}\left(r_{t}\right) w(t) \\
y(t)=C\left(r_{t}\right) x(t) \\
m(t)=M\left(r_{t}\right) x(t)+W_{2}\left(r_{t}\right) w(t) \\
x(0)=x_{0} \\
r(0)=r_{0} \\
t=0
\end{array}\right.
$$

The state observer and feedback control law for PMJSs (28) can be designed as

$$
\bar{\Sigma}_{s}^{\prime}:\left\{\begin{array}{l}
\dot{\bar{x}}=A_{i} \bar{x}(t)+B_{i} u(t)+E_{i}[m(t)-\bar{m}(t)] \\
\bar{m}(t)=M_{i} \bar{x}(t) \\
u(t)=K_{i} \bar{x}(t) \\
\bar{y}(t)=C_{i} \bar{x}(t) \\
x(0)=x_{0}, \\
r(0)=r_{0}, \\
t=0
\end{array}\right.
$$

Letting $\tilde{x}(t)=\operatorname{col}[x(t) e(t)]$, we can obtain the following closed-loop augment error dynamic MJSs:

$$
\widetilde{\Sigma}_{s}:\left\{\begin{array}{l}
\dot{\tilde{x}}=\widetilde{A}_{i} \tilde{x}(t)+\widetilde{F}_{i} f(\widetilde{x}(t), t)+\widetilde{W}_{i} w(t) \\
z(t)=\widetilde{C}_{i} \widetilde{x}(t) \\
\widetilde{x}(0)=\widetilde{x}_{0}, \\
t=0
\end{array}\right.
$$

where

$$
\begin{aligned}
\widetilde{A}_{i} & =\left[\begin{array}{cc}
A_{i}+B_{i} K_{i} & -B_{i} K_{i} \\
0 & A_{i}-E_{i} M_{i}
\end{array}\right], \\
\widetilde{F}_{i} & =\left[\begin{array}{c}
F_{1 i} \\
F_{1 i}
\end{array}\right], \\
\widetilde{W}_{i} & =\left[\begin{array}{c}
W_{1 i} \\
W_{1 i}-E_{i} W_{2 i}
\end{array}\right], \\
\widetilde{C}_{i} & =\left[\begin{array}{ll}
0 & C_{i}
\end{array}\right], \\
f(x(t), t) & =f(\widetilde{x}(t), t) .
\end{aligned}
$$

The main results in Theorem 15 will reduce to the following Corollary 19.

Corollary 19. For given constants $T>0, \mu_{1}>0, \gamma>0$, and $d>0$, there exists a finite-time $L_{2}-L_{\infty}$ observer-based control law with $K_{i}=S_{i} V_{i}^{-1}$ and $E_{i}=U_{i} M_{i}^{\mathrm{T}}$ and the augment error dynamic MJSs (29) are positive and FTS and satisfy the given $L_{2}-L_{\infty}$ disturbance rejection disturbance attenuation index (10) with regard to $\left(\mu_{1}, \mu_{2}, T, R, \gamma\right)$, where $\mu_{2}>\mu_{1}>0$ and $R>0$, if there exists a set of mode-dependent positive-definite symmetric matrices $U_{i} \in R^{n \times n}$, a set of mode-dependent matrix $S_{i} \in R^{p \times n}$, matrix $G \in R^{l \times n}$, a set of mode-dependent constants $v_{i}>0$ and $\varepsilon_{i}>0$, and constants $\mu_{2}>0, \delta>0, \lambda_{1}>0$, and $\lambda_{2}>0, \gamma>0$, such that inequalities (20)-(25) and the following relations hold:

$$
\left[\begin{array}{cccccc}
v_{1} & -B_{i} S_{i} & W_{i} U_{i} & F_{1 i} U_{i} & 0 & U_{i} G_{i}^{\mathrm{T}} \\
* & v_{2} & v_{3} & F_{1 i} U_{i} & U_{i} M_{i}^{\mathrm{T}} & 0 \\
* & * & * & -I & 0 & 0 \\
* & * & * & * & -2 I & 0 \\
* & * & * & * & * & -\beta_{i}^{-1} I
\end{array}\right]<0
$$

$$
\left[\begin{array}{ccc}
-U_{i} & 0 & 0 \\
* & -U_{i} & -C_{i}^{\mathrm{T}} \\
* & * & -\delta^{2} I
\end{array}\right]<0
$$

where $v_{1}=A_{i} U_{i}+U_{i} A_{i}^{\mathrm{T}}+B_{i} S_{i}+S_{i}^{\mathrm{T}} B_{i}^{\mathrm{T}}+\left(\pi_{i i}-\gamma\right) U_{i}, v_{2}=$ $A_{i} U_{i}+U_{i} A_{i}^{\mathrm{T}}+\left(\pi_{i i}-\gamma\right) U_{i}$, and $\nu_{3}=W_{1 i} U_{i}-M_{i}^{\mathrm{T}} W_{2 i} U_{i}$.

\section{A Numerical Example}

Consider a class of PMJSs with two modes described as follows. 
Mode 1.

$$
\begin{aligned}
A_{1} & =\left[\begin{array}{cc}
-20 & 1 \\
7 & -5
\end{array}\right], \\
B_{1} & =\left[\begin{array}{l}
1 \\
2
\end{array}\right], \\
C_{1} & =\left[\begin{array}{ll}
0.7 & 0.5
\end{array}\right], \\
L_{11} & =\left[\begin{array}{l}
0.2 \\
0.3
\end{array}\right], \\
W_{11} & =[0.1], \\
W_{21} & =[0.03], \\
F_{11} & =[0.2], \\
M_{1} & =[1], \\
G_{1} & =[0.20 .3], \\
N_{11} & =[0.40 .06], \\
N_{21} & =[0.5], \\
N_{31} & =[0.010 .03], \\
N_{41} & =[0.20 .4], \\
\alpha_{11} & =0.4, \\
\alpha_{21} & =0.2, \\
\alpha_{31} & =0.3, \\
\alpha_{41} & =0.1, \\
\beta_{1} & =1 ;
\end{aligned}
$$

Mode 2.

$$
\begin{aligned}
A_{2} & =\left[\begin{array}{cc}
-18 & 2 \\
6 & -7
\end{array}\right], \\
B_{2} & =\left[\begin{array}{l}
2 \\
1
\end{array}\right], \\
C_{2} & =\left[\begin{array}{ll}
0.05 & 0.07
\end{array}\right], \\
L_{12} & =\left[\begin{array}{l}
0.4 \\
0.1
\end{array}\right], \\
W_{12} & =\left[\begin{array}{ll}
0.02
\end{array}\right], \\
W_{22} & =\left[\begin{array}{ll}
0.05
\end{array}\right], \\
F_{12} & =\left[\begin{array}{ll}
0.04
\end{array}\right], \\
M_{2} & =\left[\begin{array}{ll}
2
\end{array}\right], \\
G_{2} & =\left[\begin{array}{ll}
0.04 & 0.03
\end{array}\right], \\
N_{12} & =\left[\begin{array}{ll}
0.1 & 0.4
\end{array}\right], \\
N_{22} & =\left[\begin{array}{ll}
0.4
\end{array}\right], \\
N_{32} & =\left[\begin{array}{ll}
0.07 & 0.04
\end{array}\right], \\
N_{42} & =\left[\begin{array}{ll}
0.8 & 0.6
\end{array}\right],
\end{aligned}
$$

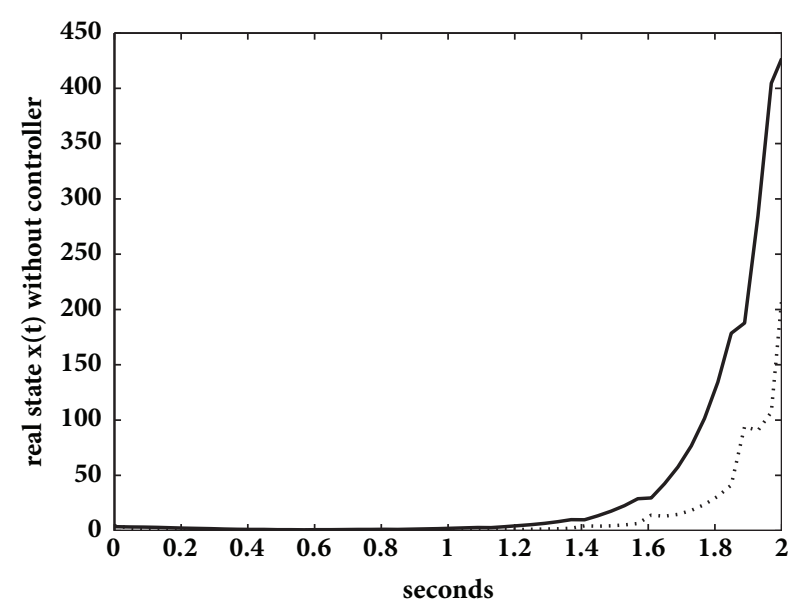

Figure 1: The plot of the jumping modes.



Figure 2: The trajectories of the real state $x(t)$ in open-loop case.

$$
\begin{aligned}
& \alpha_{12}=0.5, \\
& \alpha_{22}=0.1, \\
& \alpha_{32}=0.2, \\
& \alpha_{42}=0.4, \\
& \beta_{2}=2 .
\end{aligned}
$$

The values of the relevant parameters are given as $\gamma=$ $0.01, T=2, \lambda_{1}=1.1, \lambda_{2}=0.6228, \mu_{1}=30, \delta=2$, and $d=5$. The mode of PMJSs (2) is converted according to the following Markov chain conversion rate matrix: $\left[\begin{array}{ll}\pi_{11} & \pi_{12} \\ \pi_{21} & \pi_{22}\end{array}\right]=$ $\left[\begin{array}{cc}-2 & 2 \\ 1 & -1\end{array}\right]$ and we select the unknown nonlinear function as $f(x(t), t)=0.6 /\left(1+2 x^{2}(t)\right)$.

Solving LMIs (18)-(25) in Theorem 15, we can get the observer and the control law gain as $E_{1}=\left[\begin{array}{ll}-1.2258 & -2.4435 \\ -2.4516 & -4.8871\end{array}\right]$, $E_{2}=\left[\begin{array}{ll}-7.8209 & -4.8137 \\ -3.9104 & -2.4068\end{array}\right], K_{1}=\left[\begin{array}{ll}-1.1075 & -2.0864\end{array}\right]$, and $K_{2}=$ $[-1.0552-1.8873]$ with $\mu_{2}=37.254$.

The jumping modes, the response of the real states, the estimated state $\bar{x}(t)$, the estimated error $e(t)$, the evolution $\tilde{x}^{\mathrm{T}}(t) R \tilde{x}(t)$, and the controlled output error $z(t)$ are shown in Figures 1-6. 

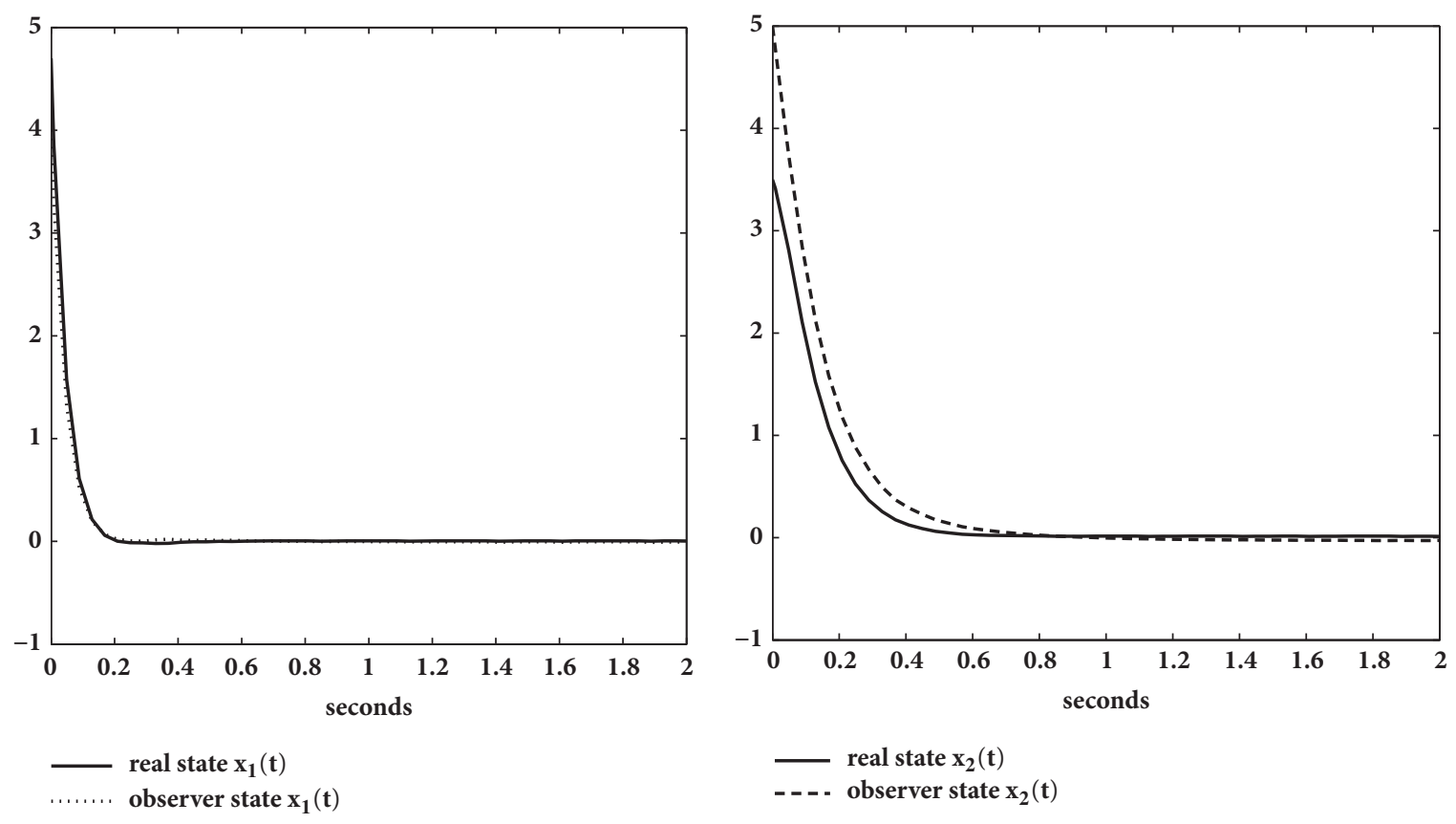

FIgURE 3: The trajectories of the real states and the observer states.

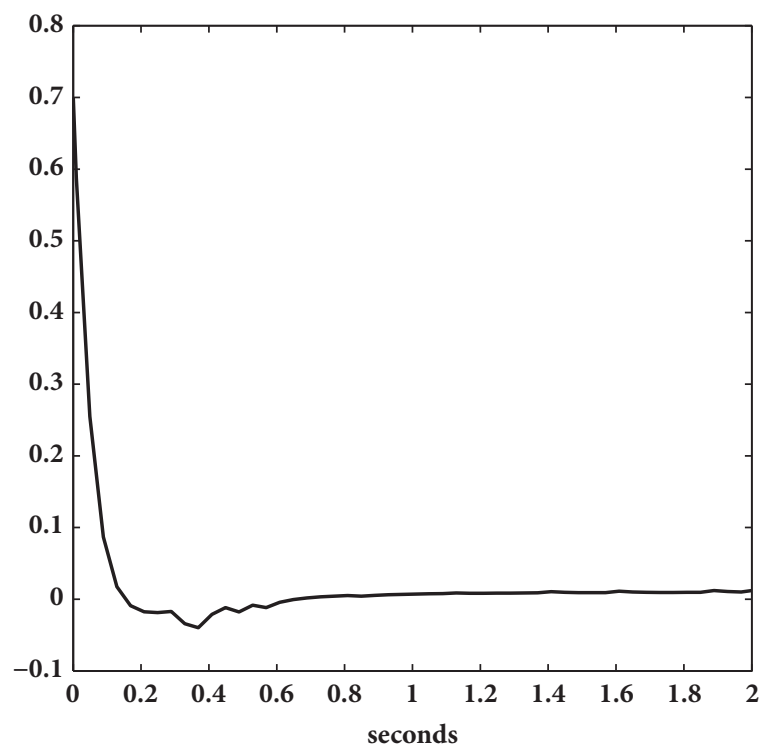

state estimate error $\mathrm{e}_{1}(\mathrm{t})$

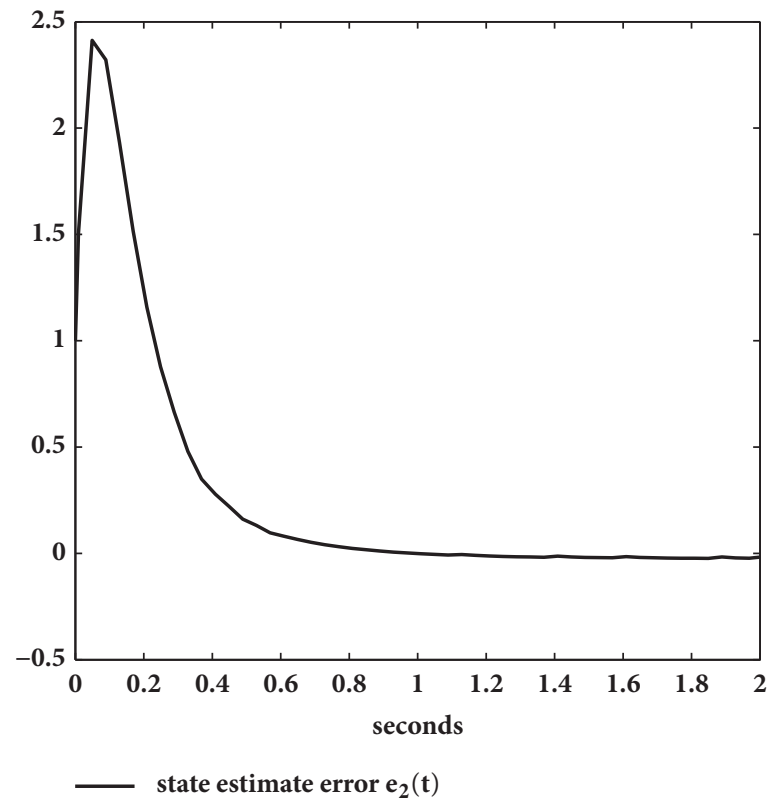

state estimate error $e_{2}(t)$

FIgURE 4: The trajectories of the state estimate errors $e(t)$.

Figure 2 gives the real state trajectory in open case and it shows that the open-loop PMJSs are unstable. The responses of the real state and the observer state are depicted in Figure 3. Figure 4 shows the state estimated error $e(t)$ and Figure 5 shows the state trajectory $\widetilde{x}^{\mathrm{T}}(t) R \widetilde{x}(t)$ of the closed-loop MJSs. From Figure 5, we know that the designed observer-based control law can ensure that the closed-loop MJSs are FTS in the given finite-time interval. Obviously, it can be seen from Figure 6 that the controlled output error of the closed-loop MJSs is positive and FTS.

\section{Conclusions}

In this paper, we studied the observer-based finite-time $L_{2}$ $L_{\infty}$ control law design problem of a class of PMJSs in a complex environment. Based on the designed SLKF methods and LMIs technique, sufficient conditions on the existence of the observer-based finite-time $L_{2}-L_{\infty}$ control law are proposed and proven. The designed finite-time $L_{2}-L_{\infty}$ control law makes the closed-loop augment error dynamic MJSs be positive and FTS and satisfy the given induced $L_{2}-L_{\infty}$ 

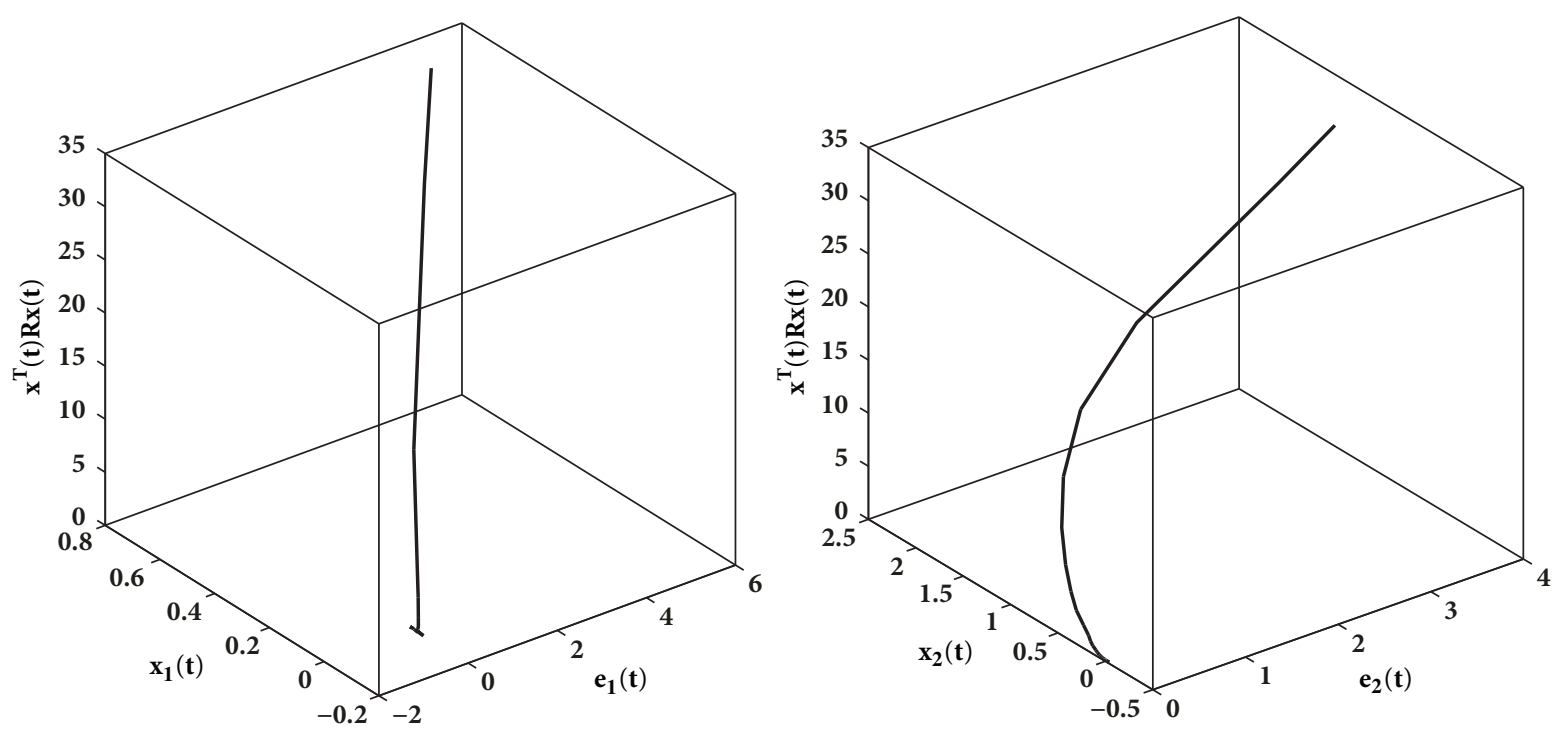

Figure 5: The evolution $\tilde{x}^{\mathrm{T}}(t) R \widetilde{x}(t)$ for $e(t)$ and $x(t)$.

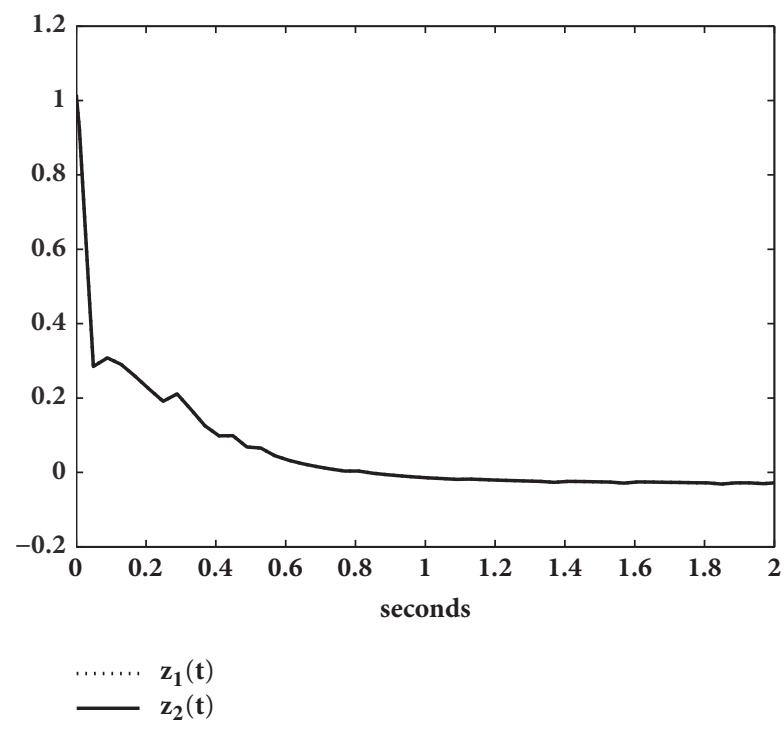

FIGURE 6: The trajectories of the controlled output errors $z(t)$.

disturbance attenuation index. A numerical example was delivered to demonstrate the contribution of the main results.

\section{Appendix}

\section{A. Proof of Theorem 13}

Proof. We select a SLKF candidate as $V[\widetilde{x}(t), i, t]=$ $\widetilde{x}^{\mathrm{T}}(t) \widetilde{P}_{i} \widetilde{x}(t)$. Recalling Definition 2 and along the trajectories of the augment error dynamic MJSs (6), the weak infinitesimal operator of $V[\widetilde{x}(t), i, t]$ can be written as

$$
\begin{aligned}
& \Im V {[\widetilde{x}(t), i, t] } \\
&= \lim _{\Delta t \rightarrow 0} \frac{1}{\Delta t}\left[\mathrm { E } \left\{V\left[\widetilde{x}(t+\Delta t), r_{(t+\Delta t)}, t+\Delta t\right] \mid \widetilde{x}(t),\right.\right. \\
&\left.\left.r_{t}\right\}-V\left[\widetilde{x}(t), r_{t}, t\right]\right]=\frac{\partial}{\partial t} V[\widetilde{x}(t), i, t]+\frac{\partial}{\partial \widetilde{x}(t)} \\
& \cdot V[\widetilde{x}(t), i, t] \cdot \dot{\tilde{x}}(t)+\sum_{j=1}^{N} \pi_{i j} V[\widetilde{x}(t), j, t] \\
&=\widetilde{x}^{\mathrm{T}}(t)\left[\left(\widetilde{A}_{i}+\widetilde{A}_{\Delta i}\right)^{\mathrm{T}} \widetilde{P}_{i}+\widetilde{P}_{i}\left(A_{i}+A_{\Delta i}\right)\right. \\
&\left.+\sum_{j=1}^{N} \pi_{i j} \widetilde{P}_{j}\right] \widetilde{x}(t)+\widetilde{x}^{\mathrm{T}}(t) \widetilde{P}_{i} \widetilde{F}_{i} f(x(t), t) \\
&+f^{\mathrm{T}}(x(t), t) F_{i}^{\mathrm{T}} \widetilde{P}_{i} \widetilde{x}(t)+\widetilde{x}^{\mathrm{T}}(t) \widetilde{P}_{i} \widetilde{W}_{i} w(t) \\
&+w^{\mathrm{T}}(t) \widetilde{W}_{i}^{\mathrm{T}} \widetilde{P}_{i} \widetilde{x}(t) .
\end{aligned}
$$

According to Lemma 9 and Assumption 10, we know that there exists a set of mode-dependent constant $\beta_{i}>0$ and matrix $G$ with proper dimension such that

$$
\begin{aligned}
f^{\mathrm{T}}( & \tilde{x}(t), t) F_{i}^{\mathrm{T}} P_{i} \widetilde{x}(t)+\widetilde{x}^{\mathrm{T}}(t) P_{i} \widetilde{F}_{i} f(\widetilde{x}(t), t) \\
\leq & \beta_{i} f^{\mathrm{T}}(\widetilde{x}(t), t) f(\widetilde{x}(t), t) \\
& +\beta_{i}^{-1} \widetilde{x}^{\mathrm{T}}(t) P_{i} \widetilde{F}_{i} \widetilde{F}_{i}^{\mathrm{T}} P_{i} \widetilde{x}(t) \\
\leq & \beta_{i} \widetilde{x}^{\mathrm{T}}(t) G^{\mathrm{T}} G \widetilde{x}(t)+\beta_{i}^{-1} \widetilde{x}^{\mathrm{T}}(t) P_{i} \widetilde{F}_{i} \widetilde{F}_{i}^{\mathrm{T}} P_{i} \widetilde{x}(t)
\end{aligned}
$$

By considering Schur complement lemma and substituting inequality (A.2) into equality (A.1), we can get

$$
\Im V[\widetilde{x}(t), i, t]=\tilde{\chi}^{\mathrm{T}}(t) \Xi_{1} \tilde{\chi}(t)
$$


where

$$
\begin{aligned}
& \tilde{\chi}(t)=\operatorname{col}\left[\begin{array}{ll}
\tilde{x}(t) & w(t)
\end{array}\right], \\
& \vartheta_{1}=\left(\widetilde{A}_{i}+\widetilde{A}_{\Delta i}\right)^{\mathrm{T}} P_{i}+P_{i}\left(A_{i}+A_{\Delta i}\right)+\beta_{i} G^{\mathrm{T}} G \\
& +\sum_{j=1}^{N} \pi_{i j} \widetilde{P}_{j}, \\
& \Xi_{1}=\left[\begin{array}{ccc}
\vartheta_{1} & \widetilde{P}_{i} \widetilde{W}_{i} & \widetilde{P}_{i} \widetilde{F}_{i} \\
* & 0 & 0 \\
* & * & -\beta_{i} I
\end{array}\right]
\end{aligned}
$$

Considering Definition 2, we introduce the following inequality:

$$
\begin{aligned}
\mathrm{E}\{\mathfrak{\Im} V[\tilde{x}(t), i, t]\}< & \gamma E[V[\tilde{x}(t), i, t]] \\
& +w(t)^{\mathrm{T}} w(t)
\end{aligned}
$$

Thus, inequality (A.5) can be obtained by inequality (15).

Multiplying inequality (A.5) by $e^{-\gamma t}$ and integrating inequality (A.5) from 0 to $t$, we can obtain

$$
\begin{aligned}
e^{-\gamma t} \mathrm{E}[V[\tilde{x}(t), i, t]]-\mathrm{E}\left[V\left[\tilde{x}(0), r_{0}, 0\right]\right] \\
<\int_{0}^{t} e^{-\gamma t} w^{\mathrm{T}}(t) w(t) d t
\end{aligned}
$$

If we define $\bar{P}_{i}=R^{-1 / 2} \widetilde{P}_{i} R^{-1 / 2}, \lambda_{1}=\max _{r \in M} \lambda_{\max }\left[\bar{P}_{i}\right]$, and $\lambda_{2}=\min _{r \in M} \lambda_{\min }\left[\bar{P}_{i}\right]$, we have the following inequality by $\gamma>0$ and $0<t<T$ :

$$
\begin{aligned}
\mathrm{E}\{V[\tilde{x}(t), i, t]\}= & \mathrm{E}\left\{\tilde{x}^{\mathrm{T}}(t) \widetilde{P}_{i} \tilde{x}(t)\right\} \\
< & e^{\gamma t} \mathrm{E}\left\{V\left[\tilde{x}_{0}, r_{0}, 0\right]\right\} \\
& +e^{\gamma t} d \int_{0}^{t} e^{-\gamma \tau} d \tau \\
& <e^{\gamma t}\left[\tilde{x}_{0}{ }^{\mathrm{T}} \widetilde{P}_{i} \tilde{x}_{0}+\frac{d}{\gamma}\left(1-e^{-\gamma t}\right)\right] \\
& <e^{\gamma t}\left[\lambda_{1} \mu_{1}+\frac{d}{\gamma}\left(1-e^{-\gamma t}\right)\right] \\
\leq & e^{\gamma T}\left[\lambda_{1} \mu_{1}+\frac{d}{\gamma}\left(1-e^{-\gamma T}\right)\right]
\end{aligned}
$$

Considering that

$$
\begin{aligned}
\mathrm{E}\{V[\tilde{x}(t), i, t]\} & =\mathrm{E}\left\{\tilde{x}^{\mathrm{T}}(t) \widetilde{P}_{i} \tilde{x}(t)\right\} \\
& \left.\geq \mathrm{E}\left\{\lambda_{2} \tilde{x}^{\mathrm{T}}(t) R \tilde{x}(t)\right]\right\}
\end{aligned}
$$

we have

$$
\mathrm{E}\left\{\lambda_{2} \widetilde{x}^{\mathrm{T}}(t) R \tilde{x}(t)\right\}<e^{\gamma T}\left[\lambda_{1} \mu_{1}+\frac{d}{\gamma}\left(1-e^{-\gamma T}\right)\right]
$$

which is equivalent to

$$
\mathrm{E}\left\{\tilde{x}^{\mathrm{T}}(t) R \tilde{x}(t)\right\}<\frac{e^{\gamma T}\left[\lambda_{1} \mu_{1}+(d / \gamma)\left(1-e^{-\gamma T}\right)\right]}{\lambda_{2}} .
$$

Therefore, $\mathrm{E}\left\{\tilde{x}^{\mathrm{T}}(t) R \tilde{x}(t)\right\}<\mu_{2}, \forall t \in\left[\begin{array}{ll}0 & T\end{array}\right]$ can be ensured through inequality (16); that is, the augment error dynamic MJSs (6) are FTS with regard to $\left(\mu_{1}, \mu_{2}, T, R, \gamma\right)$. This completes the proof.

\section{B. Proof of Theorem 14}

Proof. We select the same SLKF as Theorem 13. From inequality (A.6), we have

$$
\begin{aligned}
\mathrm{E}[V[\tilde{x}(t), i, t]]< & e^{\gamma t} \int_{0}^{t} e^{-\gamma t} w^{\mathrm{T}}(t) w(t) d t \\
& +e^{\gamma t} \mathrm{E}\left[V\left[\tilde{x}(0), r_{0}, 0\right]\right] .
\end{aligned}
$$

Under the zero initial condition, inequality (B.1) can be rewritten:

$$
\begin{aligned}
\mathrm{E}\left\{\tilde{x}^{\mathrm{T}}(t) \widetilde{P} \tilde{x}_{i}(t)\right\} & <e^{\gamma t} \int_{0}^{t} e^{-\gamma t} w^{\mathrm{T}}(t) w(t) d t \\
& <e^{\gamma T} \int_{0}^{T} e^{-\gamma t} w^{\mathrm{T}}(\tau) w(\tau) d \tau
\end{aligned}
$$

From inequality (17), it yields $\widetilde{P}_{i}-\delta^{-2} \widetilde{C}_{i}^{\mathrm{T}} \widetilde{C}_{i}>0$. Recalling Definition 3, we have

$$
\begin{aligned}
\mathrm{E}\left\{z^{\mathrm{T}}(t) z(t)\right\} & =\mathrm{E}\left\{\tilde{x}^{\mathrm{T}}(t) \widetilde{C}_{i}^{\mathrm{T}} \widetilde{C}_{i} \widetilde{x}(t)\right\} \\
& <\delta^{2} E\left\{\tilde{x}^{\mathrm{T}}(t) \widetilde{P}_{i} \widetilde{x}(t)\right\}
\end{aligned}
$$

Thus, we have $\mathrm{E}\left\{z^{\mathrm{T}}(t) z(t)\right\}<\delta^{2} e^{\gamma t} \int_{0}^{T} e^{-\gamma t} w^{\mathrm{T}}(\tau) w(\tau) d \tau$ by (B.2)-(B.3). Recalling Definition 3, we know that the finitetime $L_{2}-L_{\infty}$ disturbance rejection disturbance attenuation index (10) can be guaranteed by $\widetilde{\delta}=\delta \sqrt{e^{\gamma T}}, \forall t \in[0, T]$. This completes the proof.

\section{Proof of Theorem 15}

Proof. Considering the uncertainties in inequality (6), we substitute $\widetilde{A}_{i}, \widetilde{A}_{\Delta i}, \widetilde{F}_{i}, \widetilde{W}_{i}$, and $\widetilde{C}_{i}$ into inequalities (15) and (17) and define $\widetilde{P}_{i}=\operatorname{diag}\left\{P_{1 i} \quad P_{1 i}\right\} ;$ it yields

$$
\begin{aligned}
& \Phi_{1}+\Delta \Phi_{1}<0 \\
& \Phi_{2}+\Delta \Phi_{2}<0
\end{aligned}
$$


where

$$
\begin{aligned}
& \Phi_{1}=\left[\begin{array}{cccc}
\varphi_{11} & \varphi_{12} & \varphi_{13} & \varphi_{14} \\
* & \varphi_{22} & \varphi_{23} & \varphi_{24} \\
* & * & \varphi_{33} & 0 \\
* & * & * & \varphi_{44}
\end{array}\right], \\
& \Phi_{2}=\left[\begin{array}{ccc}
-P_{i} & 0 & 0 \\
* & -P_{i} & -C_{i}^{\mathrm{T}} \\
* & * & -\delta^{2} I
\end{array}\right], \\
& \Delta \Phi_{1}=\left[\begin{array}{cccc}
\Delta \varphi_{11} & \Delta \varphi_{12} & 0 & 0 \\
* & 0 & 0 & 0 \\
* & * & 0 & 0 \\
* & * & * & 0
\end{array}\right], \\
& \Delta \Phi_{2}=\left[\begin{array}{ccc}
0 & 0 & -C_{\Delta i}^{\mathrm{T}} \\
* & 0 & 0 \\
* & * & 0
\end{array}\right], \\
& \varphi_{11}=P_{i} A_{i}+A_{i}^{\mathrm{T}} P_{i}+P_{i} B_{i} K_{i}+K_{i}^{\mathrm{T}} B_{i}^{\mathrm{T}} P_{i}+\beta_{i} G^{\mathrm{T}} G \\
& +\sum_{j=1}^{N} \pi_{i j} P_{j}-\gamma P_{i} \\
& \varphi_{12}=-P_{i} B_{i} K_{i}, \\
& \varphi_{13}=P_{i} W_{i} \text {, } \\
& \varphi_{14}=P_{i} F_{1 i} \text {, } \\
& \varphi_{22}=P_{i} A_{i}+A_{i}^{\mathrm{T}} P_{i}+P_{i} E_{i} M_{i}+\left[P_{i} E_{i} M_{i}\right]^{\mathrm{T}} \\
& +\sum_{j=1}^{N} \pi_{i j} P_{j}-\gamma P_{i}, \\
& \varphi_{23}=P_{i} W_{1 i}-P_{i} E_{i} W_{2 i}, \\
& \varphi_{24}=P_{i} F_{1 i} \text {, } \\
& \varphi_{33}=-I \text {, } \\
& \varphi_{44}=-\beta_{i} I \text {, } \\
& \Delta \varphi_{11}=P_{i} A_{\Delta i}+A_{\Delta i}^{\mathrm{T}} P_{i}+P_{i} B_{\Delta i} K_{i}+K_{i}^{\mathrm{T}} B_{\Delta i}^{\mathrm{T}} P_{i}, \\
& \Delta \varphi_{12}=-P_{i} B_{\Delta i} K_{i}+A_{\Delta i}^{\mathrm{T}} P_{i}-M_{\Delta i}^{\mathrm{T}} E_{i}^{\mathrm{T}} P_{i} .
\end{aligned}
$$

From inequalities (C.1)-(C.2), we know that $\varphi_{22}, \varphi_{23}$, and $\Delta \varphi_{12}$ are nonlinear. Let $E_{i}=P_{i}^{-1} M_{i}^{\mathrm{T}}$ and get $\varphi_{22}=P_{i} A_{i}+$ $A_{i}^{\mathrm{T}} P_{i}+2 M_{i}^{\mathrm{T}} M_{i}+\sum_{j=1}^{N} \pi_{i j} P_{j}-\gamma P_{i}, \varphi_{23}=P_{i} W_{1 i}-M_{i}^{\mathrm{T}} W_{2 i}$, and $\Delta \varphi_{12}=-P_{i} B_{\Delta i} K_{i}+A_{\Delta i}^{\mathrm{T}} P_{i}-M_{\Delta i}^{\mathrm{T}} M_{i}$. Recalling equality (3), $\Delta \Phi_{1}$ and $\Delta \Phi_{2}$ can be rewritten as

$$
\begin{aligned}
\Delta \Phi_{1}= & \Psi_{1} \Gamma_{i}(t) \xi_{1}+\xi_{1}^{\mathrm{T}} \Gamma_{i}^{\mathrm{T}}(t) \Psi_{1}^{\mathrm{T}}+\Psi_{2} \Gamma_{i}(t) \xi_{2} \\
& +\xi_{2}^{\mathrm{T}} \Gamma_{i}^{\mathrm{T}}(t) \Psi_{1}^{\mathrm{T}}+\Psi_{3} \Gamma_{i}(t) \xi_{3}+\xi_{3}^{\mathrm{T}} \Gamma_{i}^{\mathrm{T}}(t) \Psi_{3}^{\mathrm{T}} \\
\Delta \Phi_{2}= & \Psi_{4} \Gamma_{i}(t) \xi_{4}+\xi_{4}^{\mathrm{T}} \Gamma_{i}^{\mathrm{T}}(t) \Psi_{4}^{\mathrm{T}}
\end{aligned}
$$

where $\Psi_{1}=\operatorname{col}\left[P_{i} L_{1 i} \quad 0 \quad 0 \quad 0\right], \Psi_{2}=\operatorname{col}\left[\begin{array}{llll}0 & P_{i} L_{1 i} & 0 & 0\end{array}\right]$,

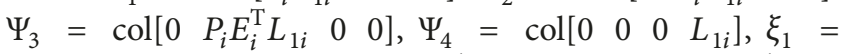
$\left[N_{1 i}+N_{2 i} K_{i}-N_{2 i} K_{i} 00\right], \xi_{2}=\left[\begin{array}{llll}N_{1 i} & 0 & 0 & 0\end{array}\right], \xi_{3}=$ $\left[\begin{array}{llll}-N_{4 i} & 0 & 0 & 0\end{array}\right]$, and $\xi_{4}=\left[\begin{array}{llll}-N_{3 i} & 0 & 0 & 0\end{array}\right]$.

According to Lemma 8, it follows from inequalities (C.1)(C.2) that

$$
\begin{aligned}
& \Phi_{1}+\alpha_{1 i}^{-1} \Psi_{1} \Psi_{1}^{\mathrm{T}}+\alpha_{1 i} \xi_{1}^{\mathrm{T}} \xi_{1}+\alpha_{2 i}^{-1} \Psi_{2} \Psi_{2}^{\mathrm{T}}+\alpha_{2 i} \xi_{2}^{\mathrm{T}} \xi_{2} \\
& +\alpha_{3 i}^{-1} \Psi_{3} \Psi_{3}^{\mathrm{T}}+\alpha_{3 i} \xi_{3}^{\mathrm{T}} \xi_{3}<0 \\
& \Phi_{2}+\alpha_{4 i}^{-1} \Psi_{4} \Psi_{4}^{\mathrm{T}}+\alpha_{4 i} \xi_{4}^{\mathrm{T}} \xi_{4}<0 .
\end{aligned}
$$

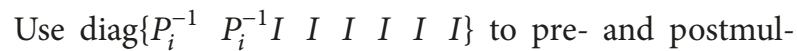
tiply matrix (C.6) and use $\operatorname{diag}\left\{P_{i}^{-1} P_{i}^{-1} I\right\}$ to pre- and postmultiply matrix (C.7). Considering $U_{i}=P_{i}^{-1}, S_{i}=$ $K_{i} P_{i}^{-1}, E_{i}=P_{i}^{-1} M_{i}^{\mathrm{T}}$, and $Q_{1 i}=M_{i}^{\mathrm{T}} P_{i}^{-1} L_{1 i} P_{i}^{-1}$ and using Schur complement lemma, we can obtain inequalities (18)(19). Noting that $\widetilde{U}_{i}=\operatorname{diag}\left\{U_{i} U_{i}\right\}$ and $\widetilde{R}=\operatorname{diag}\{R \quad R\}$, inequality (16) can be guaranteed by inequalities (20)-(21).

Next, we prove the positiveness of the augment error dynamic MJSs (6). From inequalities (22)-(23), we know that $A_{i} U_{i}+B_{i} S_{i}$ and $A_{i}-E_{i} M_{i}$ are Metzler matrices. Since $S_{i}=$ $K_{i} U_{i},\left(A_{i}+B_{i} K_{i}\right) U_{i}$ is also a Metzler matrix. From (24)-(25), we know that $-B_{i} S_{i}$ and $\left(W_{1 i}-M_{i}^{\mathrm{T}} W_{2 i}\right) U_{i}$ are positive; that is, $-B_{i} S_{i}$ and $W_{1 i}-M_{i}^{\mathrm{T}} W_{2 i}$ are positive matrices. Considering that $F_{1 i}$ is a positive matrix, $\widetilde{A}_{i}$ is a Metzler matrix and $\widetilde{F}_{i}$ and $\widetilde{W}_{i}$ are positive matrices. Recalling Lemma 6, we know that the augment error dynamic MJSs (6) are positive. This completes the proof.

\section{Data Availability}

The data findings of this study are available from the corresponding author upon request.

\section{Conflicts of Interest}

The authors declare that they have no conflicts of interest.

\section{Acknowledgments}

This work was supported in part by the National Natural Science Foundation of China under Grants 61673001 and 61203051, the Foundation for Distinguished Young Scholars of Anhui Province under Grant 1608085J05, the Key Support Program for University Outstanding Youth Talent of Anhui Province under Grant gxydZD2017001, and the open fund for Discipline Construction, Institute of Physical Science and Information Technology, Anhui University. 


\section{References}

[1] N. N. Krasovskii and È. A. Lidskiǔ, "Analytical design of controllers in systems with random attributes," Automation and Remote Control, vol. 22, pp. 1021-1025, 1961.

[2] O. L. Costa, F. E. O. Assumpcao, E. K. Boukas, and R. P. Marques, "Constrained quadratic state feedback control of discrete-time Markovian jump linear systems," Automatica, vol. 35, no. 4, pp. 617-626, 1999.

[3] N. S. D. Arrifano and V. A. Oliveira, "Robust Ho fuzzy control approach for a class of Markovian jump nonlinear systems," IEEE Transactions on Fuzzy Systems, vol. 14, no. 6, pp. 738-754, 2006.

[4] P. A. Kawka and A. G. Alleyne, "Robust wireless servo control using a discrete-time uncertain Markovian jump linear model," IEEE Transactions on Control Systems Technology, vol. 17, no. 3, pp. 733-742, 2009.

[5] S. K. Nguang, W. Assawinchaichote, and P. Shi, "Ho fuzzy filter design for uncertain nonlinear systems with Markovian jumps: an LMI approach," in Proceedings of the 2005 American Control Conference, vol. 3, pp. 1709-1804, 2015.

[6] W. Qi, J. H. Park, J. Cheng, Y. Kao, and X. Gao, "Exponential stability and L1-gain analysis for positive time-delay Markovian jump systems with switching transition rates subject to average dwell time," Information Sciences, vol. 428, pp. 24-234, 2018.

[7] Z. J. Zhao, J. Shi, X. J. Lan, X. W. Wang, and J. F. Yang, "Adaptive neural network control of a flexible string system with nonsymmetric dead-zone and output constraint," Neurocomputing, vol. 284, pp. 1-8, 2018.

[8] X. Luan, B. Huang, and F. Liu, "Higher order moment stability region for Markov jump systems based on cumulant generating function," Automatica, vol. 93, pp. 389-396, 2018.

[9] M. Hua, L. Zhang, F. Yao, J. Ni, W. Dai, and Y. Cheng, "Robust Ho filtering for continuous-time nonhomogeneous Markov jump nonlinear systems with randomly occurring uncertainties," Signal Processing, vol. 148, pp. 250-259, 2018.

[10] Y. Wang, Y. Xia, H. Shen, and P. Zhou, "SMC design for robust stabilization of nonlinear Markovian jump singular systems," IEEE Transactions on Automatic Control, vol. 63, no. 1, pp. 219224, 2018.

[11] Z. G. Wu, P. Shi, H. Su, and J. Chu, "Asynchronous L2-Lo filtering for discrete-time stochastic Markov jump systems with randomly occurred sensor nonlinearities," Automatica, vol. 50, no. 1, pp. 180-186, 2014.

[12] Z. J. Zhao, Y. Liu, and F. Guo, "Robust output feedback stabilization for a flexible marine riser system," ISA Transactions, vol. 78, pp. 130-140, 2018.

[13] H. Shen, F. Li, S. Xu, and V. Sreeram, "Slow state variables feedback stabilization for semi-markov jump systems with singular perturbations," IEEE Transactions on Automatic Control, vol. 63, no. 8, pp. 2709-2714, 2018.

[14] J. Wen, S. K. Nguang, P. Shi, and X. Zhao, "Stability and Ho control of discrete-time switched systems via one-step ahead Lyapunov function approach," IET Control Theory \& Applications, vol. 12, no. 8, pp. 1141-1147, 2018.

[15] Y. Xu, R. Lu, H. Peng, K. Xie, and A. Xue, "Asynchronous dissipative state estimation for stochastic complex networks with quantized jumping coupling and uncertain measurements," IEEE Transactions on Neural Networks and Learning Systems, vol. 28, no. 2, pp. 268-277, 2017.
[16] J. Klamka, "Positive controllability of positive dynamical systems," in Proceedings of the American Control Conference, vol. 6, pp. 616-629, 2002.

[17] T. C. Ionescu, K. Fujimoto, and J. M. A. Scherpen, "Positive and bounded real balancing for nonlinear systems - A controllability and observability function approach," in Proceedings of the 48h IEEE Conference on Decision and Control, pp. 4310-4315, December 2009.

[18] L. Farina, "On the existence of a positive realization," Systems and Control Letters, vol. 28, no. 4, pp. 219-226, 1996.

[19] B. Du, J. Lam, Z. Shu, and Y. Chen, "On reachable sets for positive linear systems under constrained exogenous inputs," Automatica, vol. 74, pp. 230-237, 2016.

[20] J. Shen and J. Lam, "Static output-feedback stabilization with optimal L1 gain for positive linear systems," Automatica, vol. 63, pp. 248-253, 2016.

[21] C. Ren and S. He, "Sliding mode control for a class of positive systems with lipschitz nonlinearities," IEEE Access, vol. 6, pp. 49811-49816, 2018.

[22] X. Liu, W. Yu, and L. Wang, "Stability analysis for continuoustime positive systems with time-varying delays," IEEE Transactions on Automatic Control, vol. 55, no. 4, pp. 1024-1028, 2010.

[23] Y. Zheng, S. S. Ge, and Y. Wang, "Non-weighted l(1) filtering for positive switched delay systems," IET Control Theory and Applications, vol. 12, no. 8, pp. 1046-1054, 2018.

[24] S. Q. Zhu, Q.-L. Han, and C. H. Zhang, "L1-gain performance analysis and positive filter design for positive discrete-time Markov jump linear systems: A linear programming approach," Automatica, vol. 50, no. 8, pp. 2098-2107, 2014.

[25] D. Zhang, Q. Zhang, and B. Lyu, "Positive state-bounding observer design for positive interval Markovian jump systems," Mathematical Problems in Engineering, vol. 2016, Article ID 2134807, 9 pages, 2016.

[26] X. Chen, J. Lam, and P. Li, "Positive filtering for continuous-time positive systems under performance," International Journal of Control, vol. 87, no. 9, pp. 1906-1913, 2014.

[27] Y. Zhang, P. Shi, S. K. Nguang, H. R. Karimi, and R. K. Agarwal, "Robust finite-time fuzzy Ho control for uncertain time-delay systems with stochastic jumps," Journal of The Franklin Institute, vol. 351, no. 8, pp. 4211-4229, 2014.

[28] S. He, J. Song, and F. Liu, "Robust finite-time bounded controller design of time-delay conic nonlinear systems using sliding mode control strategy," IEEE Transactions on Systems, Man, and Cybernetics: Systems, vol. 48, no. 11, pp. 1863-1873, 2018.

[29] C. Yang, X. Wang, Z. Li, Y. Li, and C. Su, "Teleoperation control based on combination of wave variable and neural networks," IEEE Transactions on Systems, Man, and Cybernetics: Systems, vol. 47, no. 8, pp. 2125-2136, 2017.

[30] S. He, Q. Ai, C. Ren, J. Dong, and F. Liu, "Finite-time resilient controller design of a class of uncertain nonlinear systems with time-delays under asynchronous switching," IEEE Transactions on Systems, Man, and Cybernetics: Systems, 2018.

[31] D. Zhai, L. An, X. Li, and Q. Zhang, "Adaptive fault-tolerant control for nonlinear systems with multiple sensor faults and unknown control directions," IEEE Transactions on Neural Networks and Learning Systems, vol. 29, no. 9, pp. 4436-4446, 2018.

[32] D. Zhai, L. An, D. Ye, and Q. Zhang, "Adaptive reliable Ho static output feedback control against Markovian jumping sensor failures," IEEE Transactions on Neural Networks and Learning Systems, vol. 29, no. 3, pp. 631-644, 2018. 
[33] H. Ma, H. Liang, Q. Zhu, and C. K. Ahn, "Adaptive dynamic surface control design for uncertain nonlinear strict-feedback systems with unknown control direction and disturbances," IEEE Transactions on Systems, Man, and Cybernetics: Systems, 2018.

[34] H. Ma, Q. Zhu, L. Bai, and H. Liang, "Observer-based adaptive fuzzy fault-tolerant control for stochastic nonstrict-feedback nonlinear systems with input quantization," IEEE Transactions on Neural Networks and Learning Systems, 2018.

[35] C. Yang, X. Wang, L. Cheng, and H. Ma, "Neural-learningbased telerobot control with guaranteed performance," IEEE Transactions on Cybernetics, vol. 47, no. 10, pp. 3148-3159, 2017.

[36] C. G. Yang, J. Na, G. Li, Y. N. Li, and J. P. Zhong, "Neural network for complex systems: theory and applications," Complexity, vol. 2018, Article ID 3141805, 2 pages, 2018.

[37] C. Yang, C. Zeng, C. Fang, W. He, and Z. Li, "A DMPsbased framework for robot learning and generalization of humanlike variable impedance skills," IEEE/ASME Transactions on Mechatronics, vol. 23, no. 3, pp. 1193-1203, 2018.

[38] J. Liu, L. Wei, X. Xie, E. Tian, and S. Fei, "Quantized stabilization for T-S fuzzy systems with hybrid-triggered mechanism and stochastic cyber attacks," IEEE Transactions on Fuzzy Systems, 2018.

[39] M. Syed Ali, K. Meenakshi, and N. Gunasekaran, "Finite-time $\mathrm{H} \infty$ boundedness of discrete-time neural networks normbounded disturbances with time-varying delay," International Journal of Control, Automation, and Systems, vol. 15, no. 6, pp. 2681-2689, 2017.

[40] C. Yang, Y. Jiang, W. He, J. Na, Z. Li, and B. Xu, "Adaptive Parameter Estimation and Control Design for Robot Manipulators with Finite-Time Convergence," IEEE Transactions on Industrial Electronics, vol. 65, no. 10, pp. 8112-8123, 2018.

[41] L. Farina and S. Rinaldi, Positive Linear Systems: Theory and Applications, Wiley-Interscience, New York, NY, USA, 2000. 


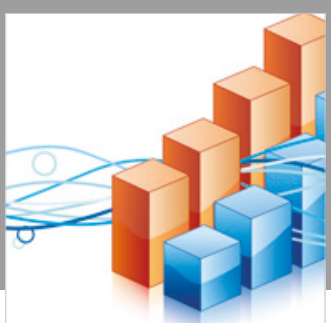

Advances in

Operations Research

\section{-n-m}
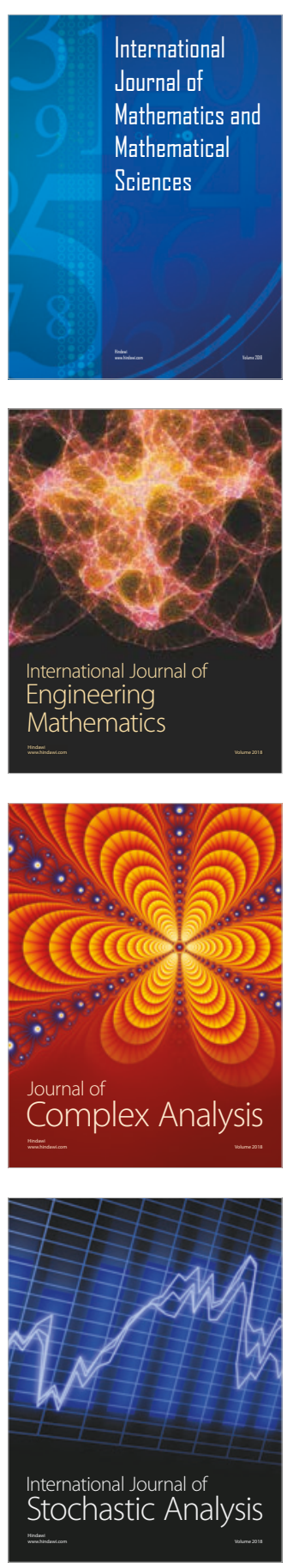
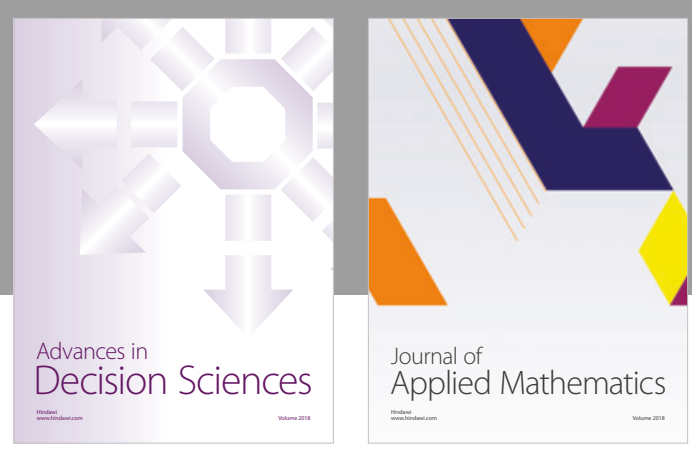

Journal of

Applied Mathematics
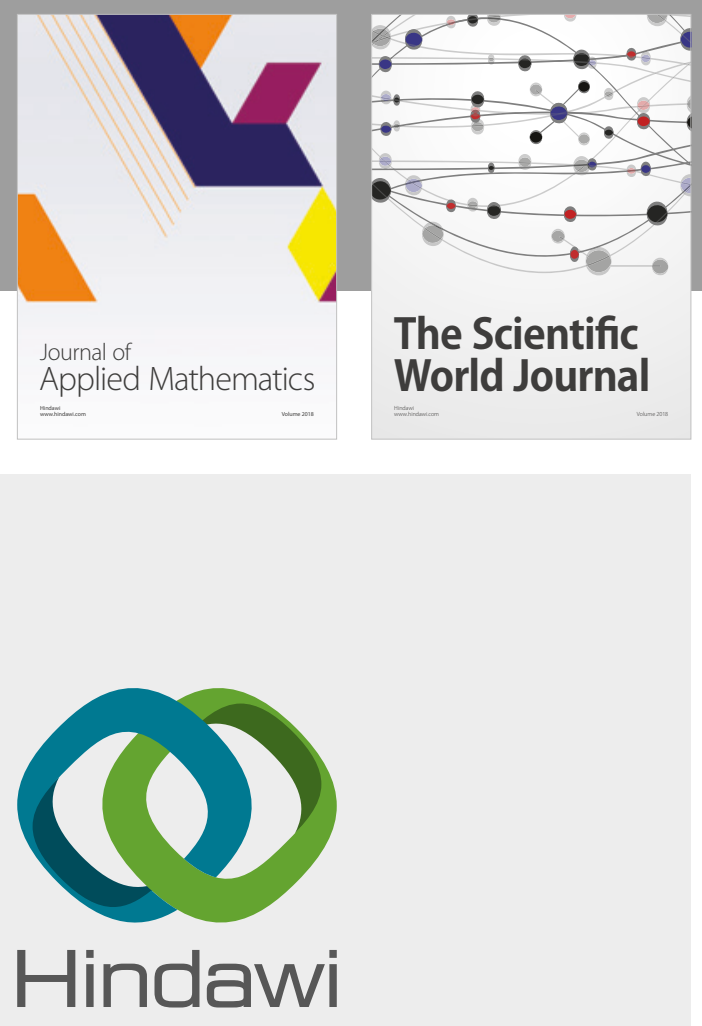

Submit your manuscripts at

www.hindawi.com

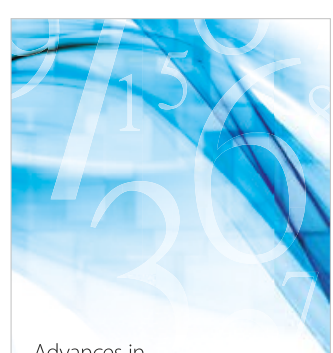

Advances in
Numerical Analysis


Mathematical Problems in Engineering

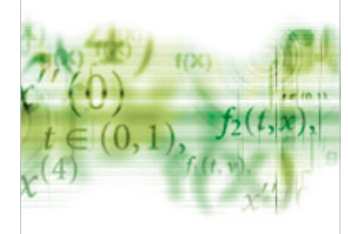

International Journal of

Differential Equations

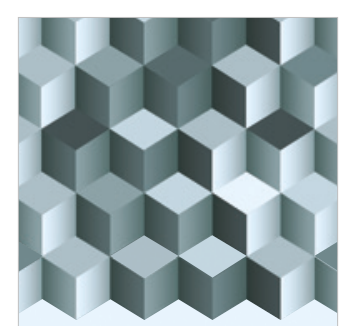

Journal of

Function Spaces
The Scientific

World Journal

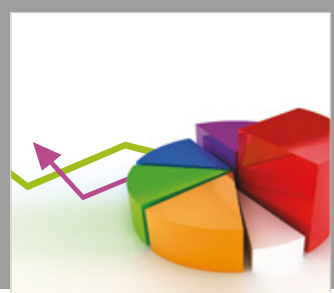

Journal of

Probability and Statistics
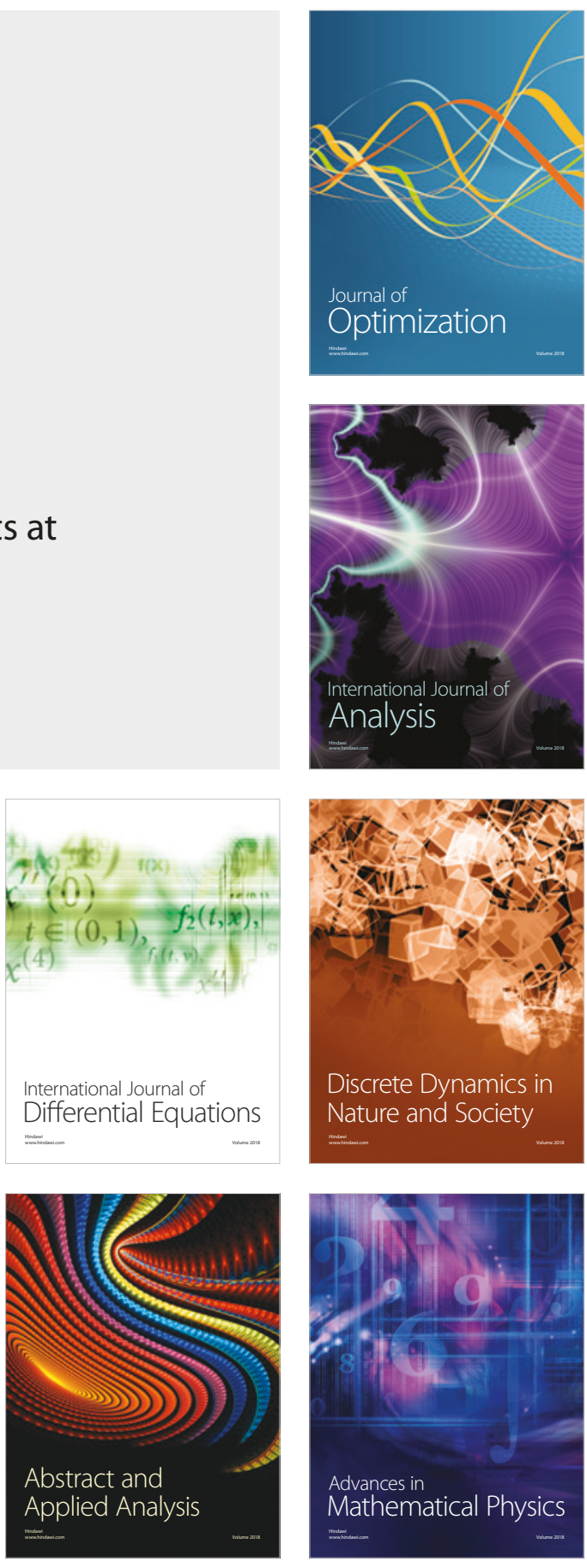